them to monitor the epidemiology of the diseases, locally as well as at national level. A weekly newsletter was also sent every Monday to all pediatricians, to inform them about the variations on the epidemiology of the infectious diseases monitored: otitis, bronchiolitis, group A Streptococcus pharyngitis, gastro-enteritis, influenza, enterovirus infections, varicella.

Conclusion The PARI network, not time-consuming for participants, based on automated data extraction from the computers of primary-care-pediatricians specially trained in infectious diseases, provides real-time data on several infectious diseases. These robust results over the past two years validate the PARI network as an efficient and reliable tool for monitoring infectious diseases and enforce the impact of this automated surveillance on the pediatricians' practice and public health.

\section{GP165 TOWARDS ESTIMATING THE INCIDENCE OF RARE DISEASES IN A PAEDIATRIC POPULATION, BORN IN IRELAND IN THE YEAR 2000}

${ }^{1}$ Emer Gunne*, ${ }^{1}$ Cliona McGarvey, ${ }^{1}$ Karina Hamilton, ${ }^{2}$ Alana Ward, ${ }^{3}$ Eileen Treacy, ${ }^{3}$ Deborah Lambert, $1,2,3$ Sally Ann Lynch. 'Temple Street Children's University Hospital, Dublin, Ireland; '2Our Lady's Children's Hospital Crumlin, Dublin, Ireland; ${ }^{3}$ Mater Misericordiae University Hospital, Dublin, Ireland

10.1136/archdischild-2019-epa.228

Background Although individually rare, (5 per 10,000) and under-recognised in healthcare systems, collectively Rare Diseases (RDs) are common, with 8,000 diseases described. There are an estimated 300,000 Rare Disease (RD) patients in Ireland; $75 \%$ of paediatric onset. The EU recognises RDs as serious, chronic, and often life threatening illnesses. The Irish National Plan for RDs (2014) recommended epidemiological studies of RD prevalence to improve both cost efficiencies and care of patients with RD's.

Objectives To derive the incidence of paediatric $\mathrm{RD}$ and the number of paediatric RD mortality cases through analysis of records held at two major tertiary paediatric hospitals, for children born in the year 2000. In addition to ascertain inhospital mortality cases with a RD and respective hospital usage.

Methods National population based study of RD patients born in Ireland in the year 2000. Cases were identified using electronic/manual records from: the National Paediatric Mortality Registry office; Clinical, Cytogenetics and Molecular genetics database; Radiology and the Hospital In-Patient Enquiry system (HIPE).

A detailed analysis of 10 years national death registration information for RDs from 2006-2016 was undertaken. In addition a study of 2 years data (2015-2016) HIPE, of RD discharges RIP age 0-14 years with analysis of their respective hospital days used.

Results There were 54,789 livebirths in 2000. Clinical, Cytogenetic and Molecular Genetics identified 603, 121 and 77 cases of RDs respectively. Ongoing HIPE searches (two major centres) identified 370 and 702 additional cases of RD. Mortality data (2006-2016) revealed 65.7\% of 105 deaths from the 2000 cohort had a RD.Of all deaths $(n=4044)$ aged 0 14, on the Register (2006-2016), 58.56\% $(n=2368)$ had a RD diagnosis with age distribution; Neonates, 56\%, Post-neonates, 58\%, Children aged 1-14 years, 64\%. Of the total $(n=365)$ 0-14 years $(2015-2016)$ inpatient deaths, $(n=234)$ $64 \%$ had a RD, 52.6\% $(n=123)$ of the deaths were at the two major centres. Of the total hospital days used by this cohort $(n=5566.5) \quad 84 \%(n=4668.5)$ of the total day usage and $77 \%(3137 / 4059)$ of ICU days used were by RD patients.

Conclusions This study has identified $>1,800$ RD patients presenting by age 17 giving a minimum incidence of $3.3 \%$ for paediatric RDs. It confirms that the use of acute hospital services by $\mathrm{RD}$ patients far exceeds that expected by their numbers. $65.7 \%$ of paediatric mortality cases for the year 2000 cohort and $58.6 \%$ of all cases from 2006-2016 had a RD confirming the serious nature of these disorders.

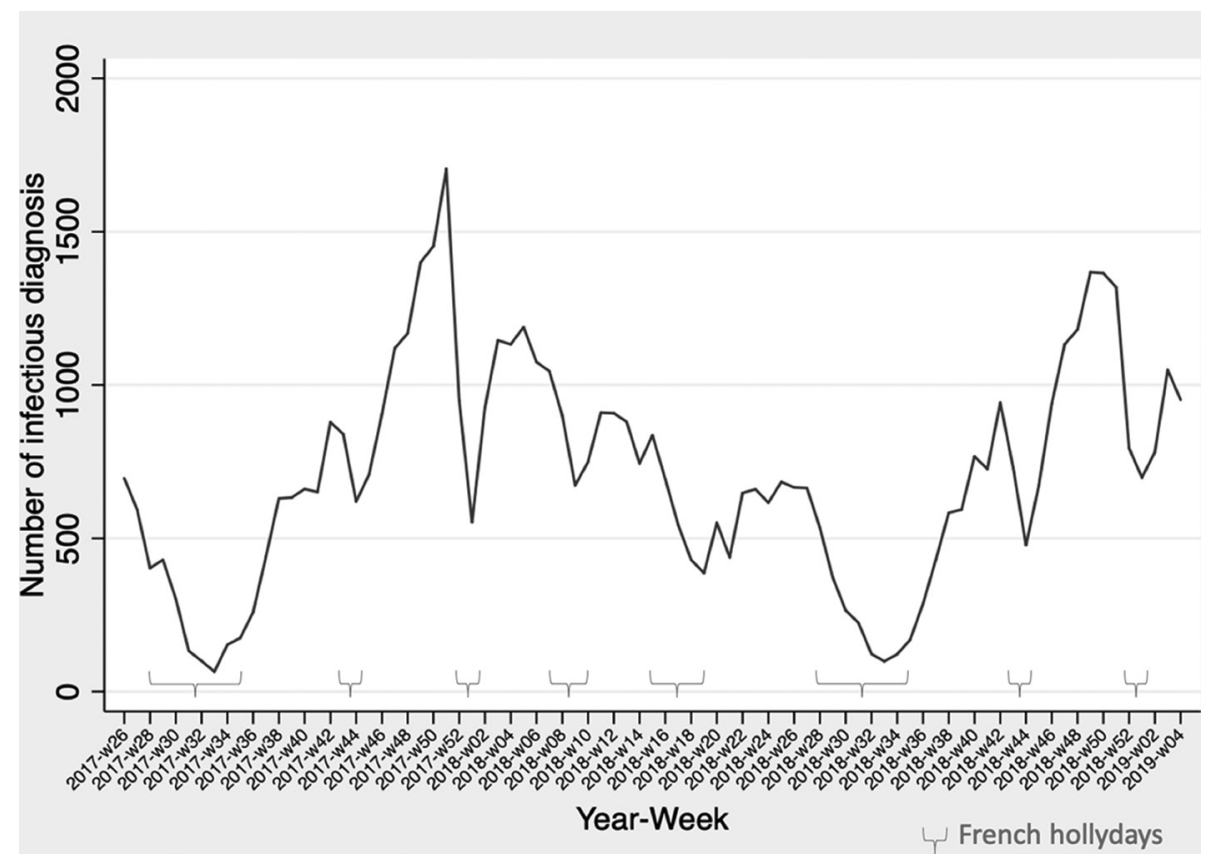

Abstract GP164 Figure 1 The PARI network 